Article

\title{
A Class of Sheffer Sequences of Some Complex Polynomials and Their Degenerate Types
}

\section{Dojin Kim}

Department of Mathematics, Pusan National University, Busan 46241, Korea; kimdojin@pusan.ac.kr

Received: 28 September 2019; Accepted: 4 November 2019; Published: 6 November 2019

check for updates

\begin{abstract}
We study some properties of Sheffer sequences for some special polynomials with complex Changhee and Daehee polynomials introducing their complex versions of the polynomials and splitting them into real and imaginary parts using trigonometric polynomial sequences. Moreover, considering their degenerate types of Sheffer sequences based on umbral composition, we present some useful expressions, properties, and examples about complex versions of the degenerate polynomials.
\end{abstract}

Keywords: Sheffer sequences; umbral calculus; binomial convolution; degeneration; Changhee polynomials; Daehee polynomials; degenerate Changhee polynomials; degenerate Daehee polynomials

\section{Introduction}

The class of Sheffer sequences is one of the most well-known classes of polynomial sequences and plays a fundamental role in solving many mathematical problems. In fact, Sheffer sequences include many important sequences: The actuarial polynomials, Frobenius-Euler polynomials, Bernoulli polynomials of the second kind, Boole polynomials, Laguerre polynomials, Bessel polynomials, Changhee polynomials, Daehee polynomials, Stirling polynomials and generalized Appell polynomials (see, for more examples, [1-7]).

For $O(f(t))=1$ and $O(g(t))=0$, all polynomials of the Sheffer sequences can be defined with the help of the following generating function (see [1,5-7] and the references cited therein)

$$
\frac{1}{g\left(f^{-1}(t)\right)} e^{x f^{-1}(t)}=\sum_{n=0}^{\infty} \mathcal{S}_{n}(x) \frac{t^{n}}{n !},
$$

where $f^{-1}(t)$ represents the compositional inverse of $f(t)$, that is, $f^{-1}(f(t))=f\left(f^{-1}(t)\right)=t$. Here, $O(f(t))$ represents the smallest integer $k$ of the non-zero power series of $f(t)$ for which the coefficient of $t^{k}$ does not vanish. The sequence of polynomials $\left\{\mathcal{S}_{n}(x)\right\}$ is called the Sheffer sequence for $(g(t), f(t))$ and is denoted by $\mathcal{S}_{n}(x) \sim(g(t), f(t))$. It is known [7] (Theorem 3.5.5) that the set of Sheffer sequences is a group of umbral composition: The identity under umbral composition is $x^{n} \sim(1, t)$ and the inverse of the sequence $\mathcal{S}_{n}(x) \sim(g(t), f(t))$ is the Sheffer sequence for $\left(\frac{1}{g\left(f^{-1}(t)\right)}, f^{-1}(t)\right)$.

Sheffer sequences for special polynomials are mainly studied by means of umbral calculus [5,7-9] and used in diverse problems of applied mathematics, theoretical physics, approximation theory, and several diverse areas of mathematics, providing significant advantages in the computational viewpoint.

In particular, after Carlitz $[10,11]$ introduced the study of the degenerate Bernoulli and Euler polynomials, the degenerate versions of special polynomials and numbers have been extensively studied by many authors in order to find useful identities and their relations (see [12-26] and references therein). The idea of the degenerate versions came from the consideration of some degenerate types 
of polynomials which can be asymptotically extended to some special generating functions such as transcendental functions $[19,27]$.

The main goal of this study is to deduce some properties and identities from the relations among complex types of Sheffer sequences of Changhee and Daehee polynomials and their degenerate types introducing their trigonometric versions. The rest of the paper is organized as follows. First, Section 2 considers two Sheffer sequences of complex Changhee and Daehee polynomials and presents some expressions and properties splitting them into real and imaginary parts with Euler's identity. Next, in Section 3, we introduce the degenerate complex Changhee and Daehee polynomials and show some useful identities by means of their trigonometric versions as done in Section 2. Finally, we summarize our conclusion in Section 4.

\section{Sheffer Sequences: Complex Changhee and Daehee Polynomials}

In this section, we first consider complex Sheffer sequences by substituting real variable $x$ with complex variable $z:=x+i y \in \mathbb{C}$ in Equation (1) and study Sheffer sequences of special complex polynomials: Changhee and Daehee polynomials.

First, by replacing $x$ with $z$ in Equation (1), we have

$$
\frac{1}{g\left(f^{-1}(t)\right)} e^{z f^{-1}(t)}=\sum_{n=0}^{\infty} \mathcal{S}_{n}(z) \frac{t^{n}}{n !}, \quad z:=x+i y \in \mathbb{C},
$$

which is complex Sheffer sequences $\left\{\mathcal{S}_{n}(z)\right\}_{n=0}^{\infty}$. We will split them into real $\Re$ and imaginary $\Im$ parts.

Definition 1. For $n \in \mathbb{N} \cup\{0\}$ and $x, y \in \mathbb{R}$, we define the cosine-Sheffer sequences $\left\{\mathcal{S}_{n}^{(c)}(x, y)\right\}_{n=0}^{\infty}$ and the sine-Sheffer sequences $\left\{\mathcal{S}_{n}^{(s)}(x, y)\right\}_{n=0}^{\infty}$ by means of the following generating functions:

$$
\begin{aligned}
& \frac{1}{g\left(f^{-1}(t)\right)} e^{x f^{-1}(t)} \cos \left(y f^{-1}(t)\right)=\sum_{n=0}^{\infty} \mathcal{S}_{n}^{(c)}(x, y) \frac{t^{n}}{n !}, \\
& \frac{1}{g\left(f^{-1}(t)\right)} e^{x f^{-1}(t)} \sin \left(y f^{-1}(t)\right)=\sum_{n=0}^{\infty} \mathcal{S}_{n}^{(s)}(x, y) \frac{t^{n}}{n !},
\end{aligned}
$$

respectively.

It is noted that the Equation (2) and Definition 1 imply that $\mathcal{S}_{n}(z)=\mathcal{S}_{n}^{(c)}(x, y)+i \mathcal{S}_{n}^{(s)}(x, y)$, that is, $\mathcal{S}_{n}^{(c)}(x, y)=\Re\left(\mathcal{S}_{n}(z)\right)$ and $\mathcal{S}_{n}^{(s)}(x, y)=\Im\left(\mathcal{S}_{n}(z)\right)$ for $n \geq 0$ and $z=x+i y \in \mathbb{C}$. Moreover, it is easily observed that

$$
\begin{aligned}
& \sum_{n=0}^{\infty} \mathcal{S}_{n}(z) \frac{t^{n}}{n !}=\frac{1}{g\left(f^{-1}(t)\right)} e^{z f^{-1}(t)}=\frac{1}{g\left(f^{-1}(t)\right)} e^{x f^{-1}(t)}\left(\cos \left(y f^{-1}(t)\right)+i \sin \left(y f^{-1}(t)\right)\right), \\
& \sum_{n=0}^{\infty} \mathcal{S}_{n}(\bar{z}) \frac{t^{n}}{n !}=\frac{1}{g\left(f^{-1}(t)\right)} e^{\bar{z} f^{-1}(t)}=\frac{1}{g\left(f^{-1}(t)\right)} e^{x f^{-1}(t)}\left(\cos \left(y f^{-1}(t)\right)-i \sin \left(y f^{-1}(t)\right)\right),
\end{aligned}
$$

which show

$$
\begin{aligned}
& \frac{1}{g\left(f^{-1}(t)\right)} e^{x f^{-1}(t)} \cos \left(y f^{-1}(t)\right)=\sum_{n=0}^{\infty}\left(\frac{\mathcal{S}_{n}(z)+\mathcal{S}_{n}(\bar{z})}{2}\right) \frac{t^{n}}{n !} \\
& \frac{1}{g\left(f^{-1}(t)\right)} e^{x f^{-1}(t)} \sin \left(y f^{-1}(t)\right)=\sum_{n=0}^{\infty}\left(\frac{\mathcal{S}_{n}(z)-\mathcal{S}_{n}(\bar{z})}{2 i}\right) \frac{t^{n}}{n !}
\end{aligned}
$$

and

$$
\mathcal{S}_{n}^{(c)}(x, y)=\frac{\mathcal{S}_{n}(z)+\mathcal{S}_{n}(\bar{z})}{2}, \quad \mathcal{S}_{n}^{(s)}(x, y)=\frac{\mathcal{S}_{n}(z)-\mathcal{S}_{n}(\bar{z})}{2 i}, \quad z=x+i y, \bar{z}=x-i y .
$$


We further investigate some properties of complex Sheffer sequences for special polynomials: Changhee polynomials and Daehee polynomials in each following subsection.

\subsection{The Complex Changhee Polynomials}

In this subsection, we study Sheffer sequences for complex Changhee polynomials $C h_{n}(z)$, originated from [6], which are given by

$$
C h_{n}(z) \sim\left(\frac{1+e^{t}}{2}, e^{t}-1\right) \quad \text { for } z=x+i y \in \mathbb{C} .
$$

Alternatively, the sequence $\left\{C h_{n}(z)\right\}_{n=0}^{\infty}$ is defined by means of the generating functions (see $[6,28,29])$ :

$$
\frac{2}{2+t}(1+t)^{z}=\sum_{n=0}^{\infty} C h_{n}(z) \frac{t^{n}}{n !}
$$

in which, when $z=0, c h_{n}=C h_{n}(0)$ are called Changhee numbers.

Note that

$$
\frac{2}{2+t}(1+t)^{x}(1+t)^{i y}=\frac{2}{2+t}(1+t)^{x}(\cos (y \log (1+t))+i \sin (y \log (1+t))),
$$

and, as shown in Definition 1, the cosine-Changhee $C h_{n}^{(c)}(x, y)$ and sine-Changhee $C h_{n}^{(s)}(x, y)$ polynomials can be respectively considered by the following generating functions:

$$
\begin{aligned}
& \frac{2}{2+t}(1+t)^{x} \cos (y \log (1+t))=\sum_{n=0}^{\infty} C h_{n}^{(c)}(x, y) \frac{t^{n}}{n !}, \\
& \frac{2}{2+t}(1+t)^{x} \sin (y \log (1+t))=\sum_{n=0}^{\infty} C h_{n}^{(s)}(x, y) \frac{t^{n}}{n !} .
\end{aligned}
$$

It is noted that $\Re\left(C h_{n}(z)\right)=C h_{n}^{(c)}(x, y)$ and $\Im\left(C h_{n}(z)\right)=C h_{n}^{(s)}(x, y)$ Moreover, one can see that the cosine-Changhee and sine-Changhee polynomials can be explicitly determined. For example, the first five consecutive polynomials can be listed:

$$
\begin{aligned}
& C h_{0}^{(c)}(x, y)=1, \quad C h_{1}^{(c)}(x, y)=x-\frac{1}{2}, \quad C h_{2}^{(c)}(x, y)=x^{2}-2 x-y^{2}+\frac{1}{2}, \\
& C h_{3}^{(c)}(x, y)=x^{3}-\frac{9}{2} x^{2}-3 x y^{2}+5 x+\frac{9}{2} y^{2}-\frac{3}{4} \\
& C h_{4}^{(c)}(x, y)=x^{4}-8 x^{3}-6 x^{2} y^{2}+20 x^{2}+24 x y^{2}-16 x+y^{4}-20 y^{2}+\frac{3}{2}
\end{aligned}
$$

and

$$
\begin{aligned}
& C h_{0}^{(s)}(x, y)=0, \quad C h_{1}^{(s)}(x, y)=y, \quad C h_{2}^{(s)}(x, y)=2 x y-2 y, \quad C h_{3}^{(s)}(x, y)=3 x^{2} y-9 x y-y^{3}+5 y, \\
& C h_{4}^{(s)}(x, y)=4 x^{3} y-24 x^{2} y-4 x y^{3}+40 x y+8 y^{3}-16 y .
\end{aligned}
$$

Now, we study their properties in detail. 
Theorem 1. Let $n$ be a nonnegative integer and $z=x+i y$. Then the real and imaginary parts of complex Changhee polynomials are expressed by

$$
\begin{aligned}
C h_{n}^{(c)}(x, y) & =\sum_{k=0}^{\left[\frac{n}{2}\right]}\left(\begin{array}{c}
n \\
2 k
\end{array}\right)(-1)^{k} y^{2 k}(\log (1+t))^{2 k} C h_{n-2 k}(x), \quad n \geq 0, \\
C h_{n}^{(s)}(x, y) & =\sum_{k=0}^{\left[\frac{n-1}{2}\right]}\left(\begin{array}{c}
n \\
2 k+1
\end{array}\right)(-1)^{k} y^{2 k+1}(\log (1+t))^{2 k+1} C h_{n-2 k-1}(x), \quad n \geq 1,
\end{aligned}
$$

where $[n]$ denotes the greatest integer less than or equal to $n$.

Proof. Considering that $\cos (y \log (1+t))=\frac{1}{2}\left(e^{i y \log (1+t)}+e^{-i y \log (1+t)}\right)$, we have

$$
\frac{2}{2+t}(1+t)^{x} \cos (y \log (1+t))=\frac{2}{2+t}(1+t)^{x} \frac{\left(e^{i y \log (1+t)}+e^{-i y \log (1+t)}\right)}{2} .
$$

Thus, using identity for the binomials convolution of sequences $\left\{C h_{n}(x)\right\}_{n=0}^{\infty}$ and $\left\{\frac{1+(-1)^{n}}{2} t^{n} \log ^{n}(1+t)\right\}_{n=0}^{\infty}$ we get identity (9) by equation (6). Similarly, one can show Equation (10) by considering $\sin (y \log (1+t))=\frac{1}{2 i}\left(e^{i y \log (1+t)}-e^{-i y \log (1+t)}\right)$.

Next, let us consider sequences of polynomials $C_{n}(x, y)$ and $S_{n}(x, y)$ given by the following generating functions:

$$
(1+t)^{x} \cos (y \log (1+t))=\sum_{n=0}^{\infty} C_{n}(x, y) \frac{t^{n}}{n !} \quad \text { and } \quad(1+t)^{x} \sin (y \log (1+t))=\sum_{n=0}^{\infty} S_{n}(x, y) \frac{t^{n}}{n !},
$$

then the sequences of polynomials $C_{n}(x, y)$ and $S_{n}(x, y)$ are explicitly determined and first few sequences can be listed as follows:

$$
\begin{aligned}
& C_{0}(x, y)=1, \quad C_{1}(x, y)=x, \quad C_{2}(x, y)=x^{2}-x-y^{2}, \quad C_{3}(x, y)=x^{3}-3 x^{2}-3 x y^{2}+2 x+3 y^{2}, \\
& C_{4}(x, y)=x^{4}-6 x^{3}-6 x^{2} y^{2}+11 x^{2}+18 x y^{2}-6 x+y^{4}-11 y^{2},
\end{aligned}
$$

and

$$
\begin{aligned}
& S_{0}(x, y)=0, \quad S_{1}(x, y)=y, \quad S_{2}(x, y)=2 x y-y, \quad S_{3}(x, y)=3 x^{2} y-6 x y-y^{3}+2 y, \\
& S_{4}(x, y)=4 x^{3} y-18 x^{2} y-4 x y^{3}+22 x y+6 y^{3}-6 y .
\end{aligned}
$$

Moreover, the following expressions for $C_{n}(x, y)$ and $S_{n}(x, y)$ are established.

Theorem 2. For $n \in \mathbb{N}$, the polynomials $C_{n}(x, y)$ and $S_{n}(x, y)$ can be expressed by

$$
\begin{aligned}
& C_{n}(x, y)=\sum_{k=0}^{n} \sum_{l=0}^{\left[\frac{k}{2}\right]} \sum_{m=0}^{n-k}\left(\begin{array}{l}
n \\
k
\end{array}\right)(-1)^{l} y^{2 l} \mathbb{S}_{1}(k, 2 l) \mathbb{S}_{1}(n-k, m) x^{m}, \quad n \geq 0, \\
& S_{n}(x, y)=\sum_{k=1}^{n} \sum_{l=0}^{\left[\frac{k-1}{2}\right]} \sum_{m=0}^{n-k}\left(\begin{array}{l}
n \\
k
\end{array}\right)(-1)^{l} y^{2 l+1} \mathbb{S}_{1}(k, 2 l+1) \mathbb{S}_{1}(n-k, m) x^{m}, \quad n>0,
\end{aligned}
$$

where $\mathbb{S}_{1}(n, m)$ are the Stirling numbers of the first kind. 
Proof. From $[6,16,30,31]$, we first recall that the Stirling numbers $\mathbb{S}_{1}(n, m)$ of the first kind satisfy

$$
\begin{aligned}
& (x)_{n}:=x(x-1) \cdots(x-n+1)=\sum_{m=0}^{n} \mathbb{S}_{1}(n, m) x^{m}, \\
& \frac{1}{m !} \log ^{m}(1+t)=\sum_{n=m}^{\infty} \mathbb{S}_{1}(n, m) \frac{t^{n}}{n !},
\end{aligned}
$$

where $(x)_{n}$ is called the falling factorial. Using Equation (12), one can show that the following equivalent expressions for the term $(1+t)^{i y}$ satisfy

$$
\begin{aligned}
(1+t)^{i y} & =e^{i y \log (1+t)} \\
& =\sum_{m=0}^{\infty} \frac{i^{m} y^{m}}{m !} \log ^{m}(1+t) \\
& =\sum_{m=0}^{\infty}(-1)^{m} y^{2 m} \frac{1}{(2 m) !} \log ^{2 m}(1+t)+i \sum_{m=0}^{\infty}(-1)^{m} y^{2 m+1} \frac{1}{(2 m+1) !} \log ^{2 m+1}(1+t) \\
& =\sum_{m=0}^{\infty}(-1)^{m} y^{2 m} \sum_{n=2 m}^{\infty} \mathbb{S}_{1}(n, 2 m) \frac{t^{n}}{n !}+i \sum_{m=0}^{\infty}(-1)^{m} y^{2 m+1} \sum_{n=2 m+1}^{\infty} \mathbb{S}_{1}(n, 2 m+1) \frac{t^{n}}{n !} \\
& =\sum_{n=0}^{\infty}\left(\sum_{m=0}^{\left[\frac{n}{2}\right]}(-1)^{m} y^{2 m} \mathbb{S}_{1}(n, 2 m)\right) \frac{t^{n}}{n !}+i \sum_{n=1}^{\infty}\left(\sum_{m=0}^{\left[\frac{n-1}{2}\right]}(-1)^{m} y^{2 m+1} \mathbb{S}_{1}(n, 2 m+1)\right) \frac{t^{n}}{n !}
\end{aligned}
$$

Similarly, one can see that

$$
(1+t)^{x}=\sum_{l=0}^{\infty} \frac{x^{l}}{l !}(\log (1+t))^{l}=\sum_{l=0}^{\infty} x^{l} \sum_{k=l}^{\infty} \mathbb{S}_{1}(k, l) \frac{t^{k}}{k !}=\sum_{k=0}^{\infty}\left(\sum_{l=0}^{k} x^{l} \mathbb{S}_{1}(k, l)\right) \frac{t^{k}}{k !}
$$

Then, by combining Equations (13) and (14), we have

$$
\begin{aligned}
(1+t)^{x}(1+t)^{i y}= & \sum_{n=0}^{\infty}\left(\sum_{k=0}^{n} \sum_{m=0}^{\left[\frac{k}{2}\right]} \sum_{l=0}^{n-k}\left(\begin{array}{l}
n \\
k
\end{array}\right)(-1)^{m} y^{2 m} \mathbb{S}_{1}(k, 2 m) \mathbb{S}_{1}(n-k, l) x^{l}\right) \frac{t^{n}}{n !} \\
& +i \sum_{n=1}^{\infty}\left(\sum_{k=1}^{n} \sum_{m=0}^{\left[\frac{k-1}{2}\right]} \sum_{l=0}^{n-k}\left(\begin{array}{l}
n \\
k
\end{array}\right)(-1)^{m} y^{2 m+1} \mathbb{S}_{1}(k, 2 m+1) \mathbb{S}_{1}(n-k, l) x^{l}\right) \frac{t^{n}}{n !}
\end{aligned}
$$

Noting the alternative expression, Equation (5), of $(1+t)^{x}(1+t)^{i y}$, it can be easily seen that the identities are satisfied from Equation (11).

Theorem 3. Let $C h_{n}^{(c)}(x, y)$ and $C h_{n}^{(s)}(x, y)$ be the cosine-Changhee polynomials and the sine-Changhee polynomials, respectively, given in Equation (6). Then, one can express these polynomials in terms of $C_{n}(x, y)$ and $S_{n}(x, y)$ in Equation (11),

$$
C h_{n}^{(c)}(x, y)=\sum_{l=0}^{n}\left(\begin{array}{l}
n \\
l
\end{array}\right) c h_{l} C_{n-l}(x, y) \quad \text { and } \quad C h_{n}^{(s)}(x, y)=\sum_{l=0}^{n}\left(\begin{array}{l}
n \\
l
\end{array}\right) c h_{l} S_{n-l}(x, y), \quad n \in \mathbb{N},
$$

respectively.

Proof. As $\frac{2}{2+t}(1+t)^{x} \cos (y \log (1+t))$ generating function for $C h_{n}^{(c)}(x, y)$, we get the first identity in the formulas by the binomial convolution of sequences $\left\{c h_{n}\right\}_{n=0}^{\infty}$ and $\left\{C_{n}(x, y)\right\}_{n=0}^{\infty}$. Similarly, the second identity can be proved. 
Theorem 4. For $n \in \mathbb{N}$, the following relations among $C h_{n}^{(c)}(x, y), C h_{n}^{(s)}(x, y), C_{n}(x, y)$, and $S_{n}(x, y)$ hold:

$$
2 C_{n}(x, y)=C h_{n}^{(c)}(x+1, y)+C h_{n}^{(c)}(x, y), \quad C_{n}(x, y)=C h_{n}^{(c)}(x, y)+\frac{n}{2} C h_{n-1}^{(c)}(x, y) \quad \forall n \geq 0,
$$

and

$$
2 S_{n}(x, y)=C h_{n}^{(s)}(x+1, y)+C h_{n}^{(s)}(x, y), \quad S_{n}(x, y)=C h_{n}^{(s)}(x, y)+\frac{n}{2} C h_{n-1}^{(s)}(x, y) \quad \forall n>0 .
$$

Proof. If we consider that

$$
\begin{aligned}
\sum_{n=0}^{\infty} C h_{n}^{(c)}(x+1, y) \frac{t^{n}}{n !} & =\frac{2}{2+t}(1+t)^{x+1} \cos (y \log (1+t)) \\
& =\frac{2}{2+t}(1+t)^{x}(2+t-1) \cos (y \log (1+t)) \\
& =2(1+t)^{x} \cos (y \log (1+t))-\frac{2}{2+t}(1+t)^{x} \cos (y \log (1+t)) \\
& =\sum_{n=0}^{\infty}\left(2 C_{n}(x, y)-C h_{n}^{(c)}(x, y)\right) \frac{t^{n}}{n !}
\end{aligned}
$$

then the first identity in Equation (17) is obtained. Next, one can also easily check the following expression:

$$
\begin{aligned}
2(1+t)^{x} \sin (y \log (1+t)) & =(2+t) \sum_{n=0}^{\infty} C h_{n}^{(s)}(x, y) \frac{t^{n}}{n !} \\
& =\sum_{n=0}^{\infty} 2 C h_{n}^{(s)}(x, y) \frac{t^{n}}{n !}+\sum_{n=1}^{\infty} n C h_{n-1}^{(s)}(x, y) \frac{t^{n}}{n !} \\
& =\sum_{n=0}^{\infty}\left(2 C h_{n}^{(s)}(x, y)+n C h_{n-1}^{(s)}(x, y)\right) \frac{t^{n}}{n !} .
\end{aligned}
$$

Alternatively, $2(1+t)^{x} \sin (y \log (1+t))=\sum_{n=0}^{\infty} 2 S_{n}(x, y) \frac{t^{n}}{n !}$, which implies the second identity in Equation (18) by comparing the coefficients in Equation (19). The remainders are similarly obtained.

\subsection{The Complex Daehee Polynomials}

In this subsection, we consider Sheffer sequences for complex Daehee polynomials $D_{n}(z)$, which are defined by

$$
D_{n}(z) \sim\left(\frac{e^{t}-1}{t}, e^{t}-1\right) \quad \text { for } z \in \mathbb{C} .
$$

Or, equivalently, the polynomials $D_{n}(z)$ are given by the generating function (see [28,30,32]):

$$
\frac{\log (1+t)}{t}(1+t)^{z}=\sum_{n=0}^{\infty} D_{n}(z) \frac{t^{n}}{n !}
$$

In particular, for $z=0, d_{n}:=D_{n}(0)$ are called Daehee numbers.

As done in the previous subsection, we also study cosine-Daehee $D_{n}^{(c)}(x, y)$ and sine-Daehee $D_{n}^{(s)}(x, y)$ polynomials defined by means of the following generating functions:

$$
\begin{aligned}
& \frac{\log (1+t)}{t}(1+t)^{x} \cos (y \log (1+t))=\sum_{n=0}^{\infty} D_{n}^{(c)}(x, y) \frac{t^{n}}{n !}, \\
& \frac{\log (1+t)}{t}(1+t)^{x} \sin (y \log (1+t))=\sum_{n=0}^{\infty} D_{n}^{(s)}(x, y) \frac{t^{n}}{n !},
\end{aligned}
$$


respectively. It is also seen that the sequences $\left\{D_{n}^{(c)}(x, y)\right\}_{n=0}^{\infty}$ and $\left\{D_{n}^{(s)}(x, y)\right\}_{n=0}^{\infty}$ satisfy $D_{n}^{(c)}(x, y)=$ $\Re\left(D_{n}(z)\right)$ and $D_{n}^{(s)}(x, y)=\Im\left(D_{n}(z)\right)$ for $z=x+i y$. They are clearly expressed and some of the first several consecutive polynomials can be listed as follows:

$$
\begin{aligned}
& D_{0}^{(c)}(x, y)=1, \quad D_{1}^{(c)}(x, y)=x-\frac{1}{2}, \quad D_{2}^{(c)}(x, y)=\frac{1}{3}\left(3 x^{2}-6 x-3 y^{2}+2\right), \\
& D_{3}^{(c)}(x, y)=(2 x-3)\left(x^{2}-3 x-3 y^{2}+1\right), \\
& D_{4}^{(c)}(x, y)=\frac{1}{5}\left(5 x^{4}-40 x^{3}-15 x^{2}\left(2 y^{2}-7\right)+20 x\left(6 y^{2}-5\right)+5 y^{4}-105 y^{2}+24\right),
\end{aligned}
$$

and

$$
\begin{aligned}
& D_{0}^{(s)}(x, y)=0, \quad D_{1}^{(s)}(x, y)=y, \quad D_{2}^{(s)}(x, y)=2 x y-2 y, \quad D_{3}^{(s)}(x, y)=3 x^{2} y-9 x y-y^{3}+\frac{11}{2} y \\
& D_{4}^{(s)}(x, y)=4 x^{3} y-24 x^{2} y-4 x y^{3}+42 x y+8 y^{3}-20 y .
\end{aligned}
$$

Theorem 5. Let $n$ be a nonnegative integer. Then the cosine- and sine-Daehee polynomials satisfy that

$$
\begin{aligned}
& D_{n}^{(c)}(x, y)=\sum_{k=0}^{\left[\frac{n}{2}\right]}\left(\begin{array}{c}
n \\
2 k
\end{array}\right)(-1)^{k} y^{2 k} \log ^{2 k}(1+t) D_{n-2 k}(x), \quad n \geq 0 \\
& D_{n}^{(s)}(x, y)=\sum_{k=0}^{\left[\frac{n-1}{2}\right]}\left(\begin{array}{c}
n \\
2 k+1
\end{array}\right)(-1)^{k} y^{2 k+1} \log ^{2 k+1}(1+t) D_{n-2 k-1}(x), \quad n \geq 1 .
\end{aligned}
$$

Proof. Recalling that $\sin (y \log (1+t))=\frac{1}{2 i}\left(e^{i y \log (1+t)}-e^{-i y \log (1+t)}\right)$, we have

$$
\frac{\log (1+t)}{t}(1+t)^{x} \sin (y \log (1+t))=\frac{\log (1+t)}{t}(1+t)^{x} \frac{\left(e^{i y \log (1+t)}-e^{-i y \log (1+t)}\right)}{2 i} .
$$

Thus, using identity for the binomials convolution of sequences $\left\{D_{n}(x)\right\}_{n=0}^{\infty}$ and $\left\{\frac{1-(-1)^{n}}{2 i} t^{n} \log ^{n}(1+t)\right\}_{n=0}^{\infty}$ we get the second identity by Equation (22). Similarly, one can show the first one by considering $\cos (y \log (1+t))=\frac{1}{2}\left(e^{i y \log (1+t)}+e^{-i y \log (1+t)}\right)$.

Theorem 6. For $n \in \mathbb{N} \cup\{0\}$, the cosine-Daehee polynomials $D_{n}^{(c)}(x, y)$ and the sine-Daehee polynomials $D_{n}^{(s)}(x, y)$ can be expressed in terms of $C_{n}(x, y)$ and $S_{n}(x, y)$ defined in Equation (11),

$$
D_{n}^{(c)}(x, y)=\sum_{l=0}^{n}\left(\begin{array}{l}
n \\
l
\end{array}\right) d_{l} C_{n-l}(x, y),(n \geq 0) \quad \text { and } \quad D_{n}^{(s)}(x, y)=\sum_{l=0}^{n}\left(\begin{array}{c}
n \\
l
\end{array}\right) d_{l} S_{n-l}(x, y),(n>0),
$$

where $d_{n}$ are Daehee numbers defined in Equation (21).

Proof. It is similarly proved as done in the proof of Theorem 3.

Theorem 7. For $n \in \mathbb{N} \cup\{0\}$, the following recursion relations of $D_{n}^{(c)}(x, y)$ and $D_{n}^{(s)}(x, y)$ hold:

$$
D_{n}^{(c)}(x+1, y)=D_{n}^{(c)}(x, y)+n D_{n-1}^{(c)}(x, y) \quad \text { and } \quad D_{n}^{(s)}(x+1, y)=D_{n}^{(s)}(x, y)+n D_{n-1}^{(s)}(x, y)
$$


Proof. We only show the first identity, since the second one can be shown in a similar way. If we consider that

$$
\begin{aligned}
\sum_{n=0}^{\infty} D_{n}^{(c)}(x+1, y) \frac{t^{n}}{n !} & =\frac{\log (1+t)}{t}(1+t)^{x+1} \cos (y \log (1+t)) \\
& =\frac{\log (1+t)}{t}(1+t)^{x}(1+t) \cos (y \log (1+t)) \\
& =\frac{\log (1+t)}{t}(1+t)^{x} \cos (y \log (1+t))+t \frac{\log (1+t)}{t}(1+t)^{x} \cos (y \log (1+t)) \\
& =\sum_{n=0}^{\infty} D_{n}^{(c)}(x, y) \frac{t^{n}}{n !}+\sum_{n=1}^{\infty} n D_{n-1}^{(c)}(x, y) \frac{t^{n}}{n !} \\
& =\sum_{n=0}^{\infty}\left(D_{n}^{(c)}(x, y)+n D_{n-1}^{(c)}(x, y)\right) \frac{t^{n}}{n !}
\end{aligned}
$$

then the first identity is obtained by the comparison of coefficients.

Corollary 1. For $n \in \mathbb{N} \cup\{0\}$, the complex Daehee polynomials $D_{n}(z)$ satisfy the recursion relation:

$$
D_{n}(z+1)=D_{n}(z)+n D_{n-1}(z) .
$$

\section{Degenerate Formulas for Sheffer Sequences of the Complex Changhee and Daehee Polynomials}

In this section, we introduce the degenerate formulas for Sheffer sequences of complex Changhee and Daehee polynomials and study their properties.

For the derivation of the degenerate formulas, for $O(f(t))=1$ and $O(g(t))=0$, we note [5] that if $\mathcal{S}_{n}(z) \sim(g(t), f(t))$ and $\mathcal{R}_{n}(z) \sim(1, l(t))$, then $\mathcal{R}_{n}(z) \circ \mathcal{S}_{n}(z) \sim(g(t), l(f(t)))$, or equivalently, we have

$$
\frac{1}{g\left(f^{-1}\left(l^{-1}(t)\right)\right)} e^{z f^{-1}\left(l^{-1}(t)\right)}=\sum_{n=0}^{\infty} \mathcal{R}_{n} \circ \mathcal{S}_{n}(z) \frac{t^{n}}{n !} .
$$

In particular, throughout this section, we will consider $l(t)=\frac{1}{\lambda} \log (1+\lambda t)$, so that $l^{-1}(t)=$ $\frac{1}{\lambda}\left(e^{\lambda t}-1\right)$ for $\lambda \in \mathbb{R} \backslash\{0\}$. Replacing $(g(t), f(t))$ by $\left(\frac{1+e^{t}}{2}, \frac{1}{\lambda} \log \left(1+\lambda\left(e^{t}-1\right)\right)\right)$ and $\left(\frac{e^{t}-1}{t}, \frac{1}{\lambda} \log (1+\right.$ $\left.\left.\lambda\left(e^{t}-1\right)\right)\right)$ for the derivation of degenerate types of complex Changee and Daehee polynomials, we have their degenerate formulas of Sheffer sequences $\left\{\mathcal{R}_{n} \circ \mathcal{S}_{n}(z)\right\}_{n=0}^{\infty}$, which will be denoted by $\left\{\mathcal{S}_{n}(z ; \lambda)\right\}_{n=0}^{\infty}$ throughout this section for the sake of convenience.

Definition 2. For $x, y \in \mathbb{R}$, we define the degenerate cosine-Sheffer sequences $\left\{\mathcal{S}_{n}^{(c)}(x, y ; \lambda)\right\}_{n=0}^{\infty}$ and the degenerate sine-Sheffer sequences $\left\{\mathcal{S}_{n}^{(s)}(x, y ; \lambda)\right\}_{n=0}^{\infty}$ by means of the following generating functions:

$$
\begin{aligned}
& \sum_{n=0}^{\infty} \mathcal{S}_{n}^{(c)}(x, y ; \lambda) \frac{t^{n}}{n !}=\frac{1}{g\left(f^{-1}\left(l^{-1}(t)\right)\right)} e^{x f^{-1}\left(l^{-1}(t)\right)} \cos \left(y f^{-1}\left(l^{-1}(t)\right)\right), \\
& \sum_{n=0}^{\infty} \mathcal{S}_{n}^{(s)}(x, y ; \lambda) \frac{t^{n}}{n !}=\frac{1}{g\left(f^{-1}\left(l^{-1}(t)\right)\right)} e^{x f^{-1}\left(l^{-1}(t)\right)} \sin \left(y f^{-1}\left(l^{-1}(t)\right)\right)
\end{aligned}
$$

respectively. 
It is noted that Equation (26) and Definition 2 imply that $\mathcal{S}_{n}(z ; \lambda)=\mathcal{S}_{n}^{(c)}(x, y ; \lambda)+i \mathcal{S}_{n}^{(s)}(x, y ; \lambda)$, that is, $\mathcal{S}_{n}^{(c)}(x, y ; \lambda)=\Re\left(\mathcal{S}_{n}(z ; \lambda)\right)$ and $\mathcal{S}_{n}^{(s)}(x, y ; \lambda)=\Im\left(\mathcal{S}_{n}(z ; \lambda)\right)$ for $n \geq 0$ and $z=x+i y \in \mathbb{C}$. Moreover, it is easily observed that

$$
\begin{aligned}
& \sum_{n=0}^{\infty} \mathcal{S}_{n}(z ; \lambda) \frac{t^{n}}{n !}=\frac{1}{g\left(f^{-1}\left(l^{-1}(t)\right)\right)} e^{x f^{-1}\left(l^{-1}(t)\right)}\left(\cos \left(y f^{-1}(t)\right)+i \sin \left(y f^{-1}(t)\right)\right), \quad z=x+i y, \\
& \sum_{n=0}^{\infty} \mathcal{S}_{n}(\bar{z} ; \lambda) \frac{t^{n}}{n !}=\frac{1}{g\left(f^{-1}\left(l^{-1}(t)\right)\right)} e^{x f^{-1}\left(l^{-1}(t)\right)}\left(\cos \left(y f^{-1}(t)\right)-i \sin \left(y f^{-1}(t)\right)\right), \quad \bar{z}=x-i y,
\end{aligned}
$$

which show

$$
\begin{aligned}
& \frac{1}{g\left(f^{-1}\left(l^{-1}(t)\right)\right)} e^{x f^{-1}\left(l^{-1}(t)\right)} \cos \left(y f^{-1}\left(l^{-1}(t)\right)\right)=\sum_{n=0}^{\infty}\left(\frac{\mathcal{S}_{n}(z ; \lambda)+\mathcal{S}_{n}(\bar{z} ; \lambda)}{2}\right) \frac{t^{n}}{n !}, \\
& \frac{1}{g\left(f^{-1}\left(l^{-1}(t)\right)\right)} e^{x f^{-1}\left(l^{-1}(t)\right)} \sin \left(y f^{-1}\left(l^{-1}(t)\right)\right)=\sum_{n=0}^{\infty}\left(\frac{\mathcal{S}_{n}(z ; \lambda)-\mathcal{S}_{n}(\bar{z} ; \lambda)}{2 i}\right) \frac{t^{n}}{n !},
\end{aligned}
$$

and

$$
\mathcal{S}_{n}^{(c)}(x, y ; \lambda)=\frac{\mathcal{S}_{n}(z ; \lambda)+\mathcal{S}_{n}(\bar{z} ; \lambda)}{2}, \quad \mathcal{S}_{n}^{(s)}(x, y ; \lambda)=\frac{\mathcal{S}_{n}(z ; \lambda)-\mathcal{S}_{n}(\bar{z} ; \lambda)}{2 i} .
$$

In the following subsections, we will consider the degenerate Sheffer sequences of the complex Changhee and Daehee polynomials $C h_{n}(z ; \lambda)$ and $D_{n}(z ; \lambda)$ given by

$$
\mathcal{S}_{n}(z ; \lambda)=C h_{n}(z ; \lambda) \sim\left(\frac{1+e^{t}}{2}, \frac{1}{\lambda} \log \left(1+\lambda\left(e^{t}-1\right)\right)\right)
$$

and

$$
\mathcal{S}_{n}(z ; \lambda)=D_{n}(z ; \lambda) \sim\left(\frac{e^{t}-1}{t}, \frac{1}{\lambda} \log \left(1+\lambda\left(e^{t}-1\right)\right)\right),
$$

respectively. We now investigate some properties of the degenerate Sheffer sequences $\left\{C h_{n}(z ; \lambda)\right\}_{n=0}^{\infty}$ and $\left\{D_{n}(z ; \lambda)\right\}_{n=0}^{\infty}$.

\subsection{Degenerate Changhee Polynomials}

In this subsection, we consider the degenerate complex Changhee polynomials $C h_{n}(z ; \lambda)$ and study some properties of them. From Equation (27), we can alternatively define $C h_{n}(z ; \lambda)$ using a generating function as follows:

Definition 3. Let us assume that $\frac{2}{2+\frac{1}{\lambda} \log (1+\lambda t)}:=\sum_{n=0}^{\infty} c h_{n, \lambda} \frac{t^{n}}{n !}$ for some sequence $\left\{c h_{n, \lambda}\right\}_{n=0}^{\infty}$. Then, for $z=x+i y \in \mathbb{C}$, we define the degenerate complex Changhee polynomials by the generating function:

$$
\frac{2}{2+\frac{1}{\lambda} \log (1+\lambda t)}\left(1+\frac{1}{\lambda} \log (1+\lambda t)\right)^{z}=\sum_{n=0}^{\infty} C h_{n}(z ; \lambda) \frac{t^{n}}{n !}, \quad \lambda \in \mathbb{R} \backslash\{0\} .
$$

In particular, when $z=0, c h_{n, \lambda}=C h_{n}(0 ; \lambda)$ are called the degenerate Changhee numbers.

For $\lambda \in \mathbb{R} \backslash\{0\}$, we consider the degenerate Euler-type formula given by

$$
\left(1+\frac{1}{\lambda} \log (1+\lambda t)\right)^{i y}:=\cos _{\lambda}^{(y)}(t)+i \sin _{\lambda}^{(y)}(t),
$$

where the degenerate cosine- and sine- functions are given by

$$
\cos _{\lambda}^{(y)}(t):=\cos \left(y \log \left(1+\frac{1}{\lambda} \log (1+\lambda t)\right)\right), \quad \sin _{\lambda}^{(y)}(t):=\sin \left(y \log \left(1+\frac{1}{\lambda} \log (1+\lambda t)\right)\right) .
$$


It is noted that $\lim _{\lambda \rightarrow 0} \sin _{\lambda}^{(y)}(t)=\sin \left(y(\log (1+t))\right.$ and $\lim _{\lambda \rightarrow 0} \cos _{\lambda}^{(y)}(t)=\cos (y \log (1+t))$.

Now, using the degenerate trigonometric Equations (31), we define the degenerate cosine-Changhee and sine-Changhee polynomials.

Definition 4. let us define the degenerate cosine-Changhee polynomials $C h_{n}^{(c)}(x, y ; \lambda)$ and the degenerate sine-Changhee polynomials $\mathrm{Ch}_{n}^{(s)}(x, y ; \lambda)$ by the generating functions, respectively, as follows:

$$
\begin{aligned}
& \frac{2}{2+\frac{1}{\lambda} \log (1+\lambda t)}\left(1+\frac{1}{\lambda} \log (1+\lambda t)\right)^{x} \cos _{\lambda}^{(y)}(t)=\sum_{n=0}^{\infty} C h_{n}^{(c)}(x, y ; \lambda) \frac{t^{n}}{n !}, \\
& \frac{2}{2+\frac{1}{\lambda} \log (1+\lambda t)}\left(1+\frac{1}{\lambda} \log (1+\lambda t)\right)^{x} \sin _{\lambda}^{(y)}(t)=\sum_{n=0}^{\infty} C h_{n}^{(s)}(x, y ; \lambda) \frac{t^{n}}{n !} .
\end{aligned}
$$

It is noted that the degenerate cosine-Changhee and sine-Changhee polynomials can be explicitly determined. For example,

$$
\begin{aligned}
C h_{0}^{(c)}(x, y ; \lambda)=1, \quad C h_{1}^{(c)}(x, y ; \lambda)=x-\frac{1}{2}, \quad C h_{2}^{(c)}(x, y ; \lambda)=-\lambda x+\frac{1}{2} \lambda+x^{2}-2 x-y^{2}+\frac{1}{2}, \\
C h_{3}^{(c)}(x, y ; \lambda)=\lambda^{2}(2 x-1)-\lambda\left(3 x^{2}-6 x-3 y^{2}+\frac{3}{2}\right)+\frac{1}{4}(2 x-3)\left(2 x^{2}-6 x-6 y^{2}+1\right), \\
C h_{4}^{(c)}(x, y ; \lambda)=\lambda^{3}(1-2 x)+\frac{11}{2} \lambda^{2}\left(2 x^{2}-4 x-2 y^{2}+1\right)-\frac{3}{2} \lambda(2 x-3)\left(2 x^{2}-6 x-6 y^{2}+1\right) \\
+x^{4}-8 x^{3}+x^{2}\left(20-6 y^{2}\right)+8 x\left(3 y^{2}-2\right)+y^{4}-20 y^{2}+\frac{3}{2}
\end{aligned}
$$

and

$$
\begin{aligned}
& C h_{0}^{(s)}(x, y ; \lambda)=0, \quad C h_{1}^{(s)}(x, y ; \lambda)=y, \quad C h_{2}^{(s)}(x, y ; \lambda)=-y(\lambda-2 x+2), \\
& C h_{3}^{(s)}(x, y ; \lambda)=y\left(-2 \lambda^{2}+6 \lambda(x-1)-3 x^{2}+9 x+y^{2}-5\right), \\
& C h_{4}^{(s)}(x, y ; \lambda)=2 y\left(-3 \lambda^{3}+11 \lambda^{2}(x-1)-6 \lambda y\left(3 x^{2}-9 x-y^{2}+5\right)+4(x-2) y\left(x^{2}-4 x-y^{2}+2\right) .\right.
\end{aligned}
$$

It is also seen that $\lim _{\lambda \rightarrow 0} C h_{n}^{(c)}(x, y ; \lambda)=C h_{n}^{(c)}(x, y)$ and $\lim _{\lambda \rightarrow 0} C h_{n}^{(s)}(x, y ; \lambda)=C h_{n}^{(s)}(x, y)$ shown in Equations (7) and (8), respectively.

Before further study of degenerate trigonometric Changhee polynomials, we recall some properties. From Equations (29)-(31) with the property (see $[6,30])$ that

$$
\frac{1}{m !}(\log (1+t))^{m}=\sum_{n=m}^{\infty} \mathbb{S}_{1}(n, m) \frac{t^{n}}{n !},
$$

we observe that

$$
\begin{aligned}
\left(1+\frac{1}{\lambda} \log (1+\lambda t)\right)^{i y} & =\sum_{k=0}^{\infty}\left(\begin{array}{c}
i y \\
k
\end{array}\right) \frac{\log ^{k}(1+\lambda t)}{\lambda^{k}} \\
& =\sum_{k=0}^{\infty}(i y)_{k} \lambda^{-k} \frac{\log ^{k}(1+\lambda t)}{k !} \\
& =\sum_{k=0}^{\infty}(i y)_{k} \lambda^{n-k} \sum_{n=k}^{\infty} \mathbb{S}_{1}(n, k) \frac{t^{n}}{n !} \\
& =\sum_{n=0}^{\infty}\left(\sum_{k=0}^{n}(i y)_{k} \lambda^{n-k} \mathbb{S}_{1}(n, k)\right) \frac{t^{n}}{n !},
\end{aligned}
$$


or alternatively,

$$
\begin{aligned}
\left(1+\frac{1}{\lambda} \log (1+\lambda t)\right)^{i y} & =e^{i y \log \left(1+\frac{1}{\lambda} \log (1+\lambda t)\right)} \\
& =\sum_{l=0}^{\infty}(i y)^{l} \frac{\log ^{l}\left(1+\frac{1}{\lambda} \log (1+\lambda t)\right)}{l !} \\
& =\sum_{l=0}^{\infty}(i y)^{l} \sum_{k=l}^{\infty} \mathbb{S}_{1}(k, l) \frac{\lambda^{-k} \log ^{k}(1+\lambda t)}{k !} \\
& =\sum_{l=0}^{\infty} i^{l} y^{l} \lambda^{-k} \sum_{k=l}^{\infty} \mathbb{S}_{1}(k, l) \sum_{n=k}^{\infty} \mathbb{S}_{1}(n, k) \frac{\lambda^{n} t^{n}}{n !} \\
& =\sum_{n=0}^{\infty}\left(\sum_{k=0}^{n} \sum_{l=0}^{k} i^{l} y^{l} \lambda^{n-k} \mathbb{S}_{1}(k, l) \mathbb{S}_{1}(n, k)\right) \frac{t^{n}}{n !}
\end{aligned}
$$

Here, from Equations (31) and (35), we directly have that

$$
\begin{aligned}
\cos _{\lambda}^{(y)}(t) & =\frac{1}{2}\left(\left(1+\frac{1}{\lambda} \log (1+\lambda t)\right)^{i y}+\left(1+\frac{1}{\lambda} \log (1+\lambda t)\right)^{-i y}\right) \\
& =\sum_{n=0}^{\infty}\left(\sum_{k=0}^{n} \sum_{l=0}^{\left[\frac{k}{2}\right]}(-1)^{l} y^{2 l} \lambda^{n-k} \mathbb{S}_{1}(k, 2 l) \mathbb{S}_{1}(n, k)\right) \frac{t^{n}}{n !}
\end{aligned}
$$

and

$$
\begin{aligned}
\sin _{\lambda}^{(y)}(t) & =\frac{1}{2 i}\left(\left(1+\frac{1}{\lambda} \log (1+\lambda t)\right)^{i y}-\left(1+\frac{1}{\lambda} \log (1+\lambda t)\right)^{-i y}\right) \\
& =\sum_{n=1}^{\infty}\left(\sum_{k=1}^{n} \sum_{l=0}^{\left[\frac{k-1}{2}\right]}(-1)^{l} y^{2 l+1} \lambda^{n-k} \mathbb{S}_{1}(k, 2 l+1) \mathbb{S}_{1}(n, k)\right) \frac{t^{n}}{n !}
\end{aligned}
$$

From the properties from Equations (34) to (37), we have the following:

Theorem 8. For $n \geq 0$ and $z=x+i y$, the following identities of the degenerate Changhee polynomials are satisfied:

$$
\begin{aligned}
C h_{n}(z ; \lambda) & =\sum_{k=0}^{n} \sum_{l=0}^{k}\left(\begin{array}{l}
n \\
k
\end{array}\right) C h_{n-k}(x ; \lambda)(i y)_{l} \lambda^{k-l} \mathbb{S}_{1}(k, l) \\
& =\sum_{k=0}^{n} \sum_{l=0}^{k}\left(\begin{array}{l}
n \\
k
\end{array}\right) c h_{n-k, \lambda}(z)_{l} \lambda^{k-l} \mathbb{S}_{1}(k, l) .
\end{aligned}
$$

Proof. As $\frac{2}{2+\frac{1}{\lambda} \log (1+\lambda t)}\left(1+\frac{1}{\lambda} \log (1+\lambda t)\right)^{z}$ generating functions for $C h_{n}(z ; \lambda)$, we have directly the first identity by the binomial convolution of sequences $\left\{C h_{n}(x ; \lambda)\right\}_{n=0}^{\infty}$ and $\left\{\sum_{k=0}^{n}(i y)_{k} \lambda^{n-k} \mathbb{S}_{1}(n, k)\right\}_{n=0}^{\infty}$ by Equation (34). On the other hand, by replacing iy by $z$ in Equation (34), we get the second identity by the binomial convolution of sequences $\left\{c h_{n}\right\}_{n=0}^{\infty}$ and $\left\{\sum_{k=0}^{n}(z)_{k} \lambda^{n-k} \mathbb{S}_{1}(n, k)\right\}_{n=0}^{\infty}$.

Theorem 9. Let us consider the following generating functions given by

$$
\begin{aligned}
& \left(1+\frac{1}{\lambda} \log (1+\lambda t)\right)^{x} \cos _{\lambda}^{(y)}(t)=\sum_{n=0}^{\infty} C_{n}(x, y ; \lambda) \frac{t^{n}}{n !}, \\
& \left(1+\frac{1}{\lambda} \log (1+\lambda t)\right)^{x} \sin _{\lambda}^{(y)}(t)=\sum_{n=0}^{\infty} S_{n}(x, y ; \lambda) \frac{t^{n}}{n !}
\end{aligned}
$$


for some polynomials $C_{n}(x, y ; \lambda)$ and $S_{n}(x, y ; \lambda)$, then the polynomials can be expressed by

$$
\begin{aligned}
& C_{n}(x, y ; \lambda)=\sum_{m=0}^{n} \sum_{k=0}^{m} \sum_{j=0}^{\left[\frac{k}{2}\right]} \sum_{l=0}^{n-m}\left(\begin{array}{c}
n \\
m
\end{array}\right)(x)_{l} \lambda^{n-k-l}(-1)^{j} y^{2 j} \mathbb{S}_{1}(n-m, l) \mathbb{S}_{1}(m, k) \mathbb{S}_{1}(k, 2 j), n \geq 0, \\
& S_{n}(x, y ; \lambda)=\sum_{m=1}^{n} \sum_{k=1}^{m} \sum_{j=0}^{\left[\frac{k-1}{2}\right]} \sum_{l=0}^{n-m}\left(\begin{array}{c}
n \\
m
\end{array}\right)(x)_{l} \lambda^{n-k-l}(-1)^{j} y^{2 j+1} \mathbb{S}_{1}(n-m, l) \mathbb{S}_{1}(m, k) \mathbb{S}_{1}(k, 2 j+1), n \geq 1,
\end{aligned}
$$

where $(x)_{n}$ and $\mathbb{S}_{1}(n, m)$ are the falling factorial and the Stirling numbers of the first kind, respectively.

Proof. Noting that $\left(1+\frac{1}{\lambda} \log (1+\lambda t)\right)^{x}=\sum_{n=0}^{\infty}\left(\sum_{l=0}^{n}(x)_{l} \lambda^{n-l} \mathbb{S}_{1}(n, l)\right) \frac{t^{n}}{n !}$, one can easily obtain the results by the binomial product of Equations (36) and (37), respectively.

We next give an expression of $C h_{n}^{(c)}(x, y ; \lambda)$ and $C h_{n}^{(s)}(x, y ; \lambda)$ in terms of Stirling numbers of the first kind.

Theorem 10. For $n \in \mathbb{N} \cup\{0\}$, the identities of the degenerate cosine- and sine-Changhee polynomials are established:

$$
\begin{aligned}
& C h_{n}^{(c)}(x, y ; \lambda)=\sum_{k=0}^{n} \sum_{m=0}^{\left[\frac{k}{2}\right]}\left(\begin{array}{l}
n \\
k
\end{array}\right) C h_{n-k}(x ; \lambda)(-1)^{m} y^{2 m} \lambda^{n-k} \mathbb{S}_{1}(k, 2 m) \mathbb{S}_{1}(n, k) \quad \text { for } n \geq 0, \\
& C h_{n}^{(s)}(x, y ; \lambda)= \begin{cases}0, & \text { when } n=0, \\
\sum_{k=1}^{n} \sum_{m=0}^{\left[\frac{k-1}{2}\right]}\left(\begin{array}{l}
n \\
k
\end{array}\right) C h_{n-k}(x ; \lambda)(-1)^{m} y^{2 m+1} \lambda^{n-k} \mathbb{S}_{1}(k, 2 m+1) \mathbb{S}_{1}(n, k), & \text { when } n>0 .\end{cases}
\end{aligned}
$$

Proof. As $\frac{2}{2+\frac{1}{\lambda} \log (1+\lambda t)}\left(1+\frac{1}{\lambda} \log (1+\lambda t)\right)^{x} \cos _{\lambda}^{(y)}(t)$ generating functions for $C h_{n}^{(c)}(x, y ; \lambda)$, from Equation (36), we have directly the first identity by the binomial convolution of sequences:

$$
\left\{C h_{n}(x ; \lambda)\right\}_{n=0}^{\infty} \text { and }\left\{\sum_{k=0}^{n} \sum_{m=0}^{\left[\frac{k}{2}\right]}(-1)^{m} y^{2 m} \lambda^{n-k} \mathbb{S}_{1}(k, 2 m) \mathbb{S}_{1}(n, k)\right\}_{n=0}^{\infty}
$$

In a similar way, as $\frac{2}{2+\frac{1}{\lambda} \log (1+\lambda t)}\left(1+\frac{1}{\lambda} \log (1+\lambda t)\right)^{x} \sin _{\lambda}^{(y)}(t)$ generating functions for $C h_{n}^{(s)}(x, y ; \lambda)$, by Equation (37), we get the second identity by the binomial convolution of sequences:

$$
\left\{C h_{n}(x ; \lambda)\right\}_{n=0}^{\infty} \text { and }\left\{\sum_{k=1}^{n} \sum_{m=0}^{\left[\frac{k-1}{2}\right]}(-1)^{m} y^{2 m+1} \lambda^{n-k} \mathbb{S}_{1}(k, 2 m+1) \mathbb{S}_{1}(n, k)\right\}_{n=1}^{\infty}
$$

Theorem 11. For $n \geq 0, z=x+i y$, and $r \in \mathbb{R}$, the polynomials $C h_{n}^{(c)}(x, y ; \lambda)$ and $C h_{n}^{(s)}(x, y ; \lambda)$ satisfy that

$$
\begin{aligned}
& C h_{n}^{(c)}(x+r, y ; \lambda)=\sum_{k=0}^{n} \sum_{l=0}^{k}\left(\begin{array}{l}
n \\
k
\end{array}\right) C h_{n-k}^{(c)}(x, y ; \lambda)(r)_{l} \lambda^{k-l} \mathbb{S}_{1}(k, l), \quad n \geq 0, \\
& C h_{n}^{(s)}(x+r, y ; \lambda)=\sum_{k=0}^{n} \sum_{l=0}^{k}\left(\begin{array}{l}
n \\
k
\end{array}\right) C h_{n-k}^{(s)}(x, y ; \lambda)(r)_{l} \lambda^{k-l} \mathbb{S}_{1}(k, l), \quad n \geq 1 .
\end{aligned}
$$


Proof. We first note that for $r \in \mathbb{R}$

$$
\left(1+\frac{1}{\lambda} \log (1+\lambda t)\right)^{r}=\sum_{n=0}^{\infty}\left(\sum_{l=0}^{n}(r){ }_{l} \lambda^{n-l} \mathbb{S}_{1}(n, l)\right) \frac{t^{n}}{n !} .
$$

As $\frac{2}{2+\frac{1}{\lambda} \log (1+\lambda t)}\left(1+\frac{1}{\lambda} \log (1+\lambda t)\right)^{x+r} \cos _{\lambda}^{(y)}(t)$ generating function for $C h_{n}^{(c)}(x+r, y ; \lambda)$, we get the first identity by the binomial convolution of sequences $\left\{C h_{n}^{(c)}(x, y ; \lambda)\right\}_{n=0}^{\infty}$ and $\left\{\sum_{k=0}^{n}(r)_{k} \lambda^{n-k} \mathbb{S}_{1}(n, k)\right\}_{n=0}^{\infty}$. Similarly, the second identity can be proved.

Theorem 12. For $n \in \mathbb{N} \cup\{0\}$, the following relations among $C h_{n}^{(c)}(x, y ; \lambda), C h_{n}^{(s)}(x, y ; \lambda), C_{n}(x, y ; \lambda)$, and $S_{n}(x, y ; \lambda)$ hold:

$$
\begin{aligned}
2 C_{n}(x, y ; \lambda)=C h_{n}^{(c)}(x+1, y ; \lambda)+C h_{n}^{(c)}(x, y ; \lambda), & 2 S_{n}(x, y ; \lambda)=C h_{n}^{(s)}(x+1, y ; \lambda)+C h_{n}^{(s)}(x, y ; \lambda) \quad \forall n \geq 0, \\
C_{n}(x, y ; \lambda)=C h_{n}^{(c)}(x, y ; \lambda)+\frac{n}{2} C h_{n-1}^{(c)}(x, y ; \lambda), & S_{n}(x, y ; \lambda)=C h_{n}^{(s)}(x, y ; \lambda)+\frac{n}{2} C h_{n-1}^{(s)}(x, y ; \lambda) \quad \forall n>0 .
\end{aligned}
$$

Proof. The proof is similar to the one in Theorem 4.

Corollary 2. For $n \in \mathbb{N}$, the following relations are satisfied:

$$
\begin{aligned}
& 2 C_{n}(z ; \lambda)+i 2 S_{n}(z ; \lambda)=C h_{n}(z+1 ; \lambda)+C h_{n}(z ; \lambda) \quad \forall n \geq 0, \\
& C_{n}(z ; \lambda)+i S_{n}(z ; \lambda)=C h_{n}(z ; \lambda)+\frac{n}{2} C h_{n-1}(z ; \lambda) \quad \forall n>0 .
\end{aligned}
$$

\subsection{Degenerate Daehee Polynomials}

In this subsection, we study some properties of Sheffer sequences for the degenerate complex Daehee polynomials. First, we can explicitly define $D_{n}(z ; \lambda)$, from Equation (28), as follows:

Definition 5. For $z=x+i y$ and $\lambda \in \mathbb{R} \backslash\{0\}$, we define the degenerate complex Daehee polynomials $D_{n}(z ; \lambda)$ by the generating function:

$$
\frac{\log (1+\lambda \log (1+t))}{\log (1+\lambda t)}\left(1+\frac{1}{\lambda} \log (1+\lambda t)\right)^{z}=\sum_{n=0}^{\infty} D_{n}(z ; \lambda) \frac{t^{n}}{n !}
$$

In particular, $d_{n, \lambda}:=D_{n}(0 ; \lambda)$ are called the degenerate Daehee numbers. It is noted that $\frac{\log (1+\lambda \log (1+t))}{\log (1+\lambda t)}=\sum_{n=0}^{\infty} d_{n, \lambda} \frac{t^{n}}{n !}$ and $\lim _{\lambda \rightarrow 0} \frac{\log (1+\lambda \log (1+t))}{\log (1+\lambda t)}=\frac{\log (1+t)}{t}$.

With the help of the degenerate trigonometric functions defined in Equation (31), we define the degenerate trigonometric Daehee polynomials.

Definition 6. For $z=x+i y$, let us define the degenerate cosine-Daehee polynomials $D_{n}^{(c)}(x, y ; \lambda)$ and the degenerate sine-Daehee polynomials $D_{n}^{(s)}(x, y ; \lambda)$ by the generating functions, respectively, as follows:

$$
\begin{aligned}
& \frac{\log (1+\lambda \log (1+t))}{\log (1+\lambda t)}\left(1+\frac{1}{\lambda} \log (1+\lambda t)\right)^{x} \cos _{\lambda}^{(y)}(t)=\sum_{n=0}^{\infty} D_{n}^{(c)}(x, y ; \lambda) \frac{t^{n}}{n !}, \\
& \frac{\log (1+\lambda \log (1+t))}{\log (1+\lambda t)}\left(1+\frac{1}{\lambda} \log (1+\lambda t)\right)^{x} \sin _{\lambda}^{(y)}(t)=\sum_{n=0}^{\infty} D_{n}^{(s)}(x, y ; \lambda) \frac{t^{n}}{n !},
\end{aligned}
$$

respectively. 
We note that $\Re D_{n}(z ; \lambda)=D_{n}^{(c)}(x, y ; \lambda)$ and $\Im D_{n}(z ; \lambda)=D_{n}^{(s)}(x, y ; \lambda)$ and the degenerate cosine-Daehee and sine-Daehee polynomials can be explicitly determined and some of them are listed:

$$
\begin{aligned}
D_{0}^{(c)}(x, y ; \lambda)= & 1, \quad D_{1}^{(c)}(x, y ; \lambda)=x-\frac{1}{2}, \quad D_{2}^{(c)}(x, y ; \lambda)=\lambda\left(\frac{1}{2}-x\right)+x^{2}-2 x-y^{2}+\frac{2}{3}, \\
D_{3}^{(c)}(x, y ; \lambda)= & \lambda^{2}\left(4 x-\frac{5}{2}\right)+\lambda\left(-6 x^{2}+12 x+y^{2}-\frac{7}{2}\right)+(2 x-3)\left(x^{2}-3 x-3 y^{2}+1\right), \\
D_{4}^{(c)}(x, y ; \lambda)= & -\frac{3}{2} \lambda^{3}(4 x-3)+\frac{1}{6} \lambda^{2}\left(66 x^{2}-138 x-66 y^{2}+47\right)-\lambda\left(6 x^{3}-27 x^{2}+x\left(32-18 y^{2}\right)\right) \\
& +\lambda\left(27 y^{2}-7\right)+x^{4}-8 x^{3}-3 x^{2}\left(2 y^{2}-7\right)+4 x\left(6 y^{2}-5\right)+y^{4}-21 y^{2}+\frac{24}{5},
\end{aligned}
$$

and

$D_{0}^{(s)}(x, y ; \lambda)=0, \quad D_{1}^{(s)}(x, y ; \lambda)=y, \quad D_{2}^{(s)}(x, y ; \lambda)=y(-\lambda+2 x-2)$,

$D_{3}^{(s)}(x, y ; \lambda)=y\left(2 \lambda^{2}-6 \lambda(x-1)+3 x^{2}-9 x-y^{2}+\frac{11}{2}\right)$

$D_{4}^{(s)}(x, y ; \lambda)=y\left(-6 \lambda^{3}+\lambda^{2}(22 x-23)+\lambda\left(-18 x^{2}+54 x+6 y^{2}-32\right)\right)+2(x-2) y\left(2 x^{2}-8 x-2 y^{2}+5\right)$,

where one can see that $\lim _{\lambda \rightarrow 0} D_{n}^{(s)}(x, y ; \lambda)=D_{n}^{(s)}(x, y)$ and $\lim _{\lambda \rightarrow 0} D_{n}^{(c)}(x, y ; \lambda)=D_{n}^{(c)}(x, y)$ for $n=0, \cdots, 4$, (see Equations (23) and (24)).

Theorem 13. For $n \geq 0$ and $z=x+i y$, the following identities are satisfied:

$$
\begin{aligned}
D_{n}(z ; \lambda) & =\sum_{k=0}^{n} \sum_{l=0}^{k}\left(\begin{array}{l}
n \\
k
\end{array}\right) D_{n-k}(x ; \lambda)(i y)_{l} \lambda^{k-l} \mathbb{S}_{1}(k, l) \\
& =\sum_{k=0}^{n} \sum_{l=0}^{k}\left(\begin{array}{l}
n \\
k
\end{array}\right) d_{n-k, \lambda}(x+i y)_{l} \lambda^{k-l} \mathbb{S}_{1}(k, l),
\end{aligned}
$$

where $d_{n, \lambda}$ are the degenerate Daehee numbers given in Definition 5.

Proof. Since $\frac{\log (1+\lambda \log (1+t))}{\log (1+\lambda t)}\left(1+\frac{1}{\lambda} \log (1+\lambda t)\right)^{x}\left(1+\frac{1}{\lambda} \log (1+\lambda t)\right)^{i y}$ is the generating function for $D_{n}(z ; \lambda)$, using Equation (34) we get the first identity by the binomial convolution of sequences:

$$
\left\{D_{n}(x ; \lambda)\right\}_{n=0}^{\infty} \text { and }\left\{\sum_{k=0}^{n}(i y)_{k} \lambda^{n-k} \mathbb{S}_{1}(n, k)\right\}_{n=0}^{\infty} .
$$

Similarly, the second identity can be proved by the binomial convolution of sequences:

$$
\left\{d_{n, \lambda}\right\}_{n=0}^{\infty} \quad \text { and } \quad\left\{\sum_{k=0}^{n}(x+i y)_{k} \lambda^{n-k} \mathbb{S}_{1}(n, k)\right\}_{n=0}^{\infty}
$$

Theorem 14. For $n \in \mathbb{N} \cup\{0\}$ and $x=x+i y$, the degenerate cosine- and sine-Daehee polynomials satisfy that

$$
\begin{aligned}
& D_{n}^{(c)}(x, y ; \lambda)=\sum_{k=2 m}^{n} \sum_{m=0}^{\left[\frac{k}{2}\right]}\left(\begin{array}{l}
n \\
k
\end{array}\right) D_{n-k}(x ; \lambda)(-1)^{m} y^{2 m} \lambda^{k} \mathbb{S}_{1}(k, 2 m) \quad \text { for } n \geq 0, \\
& D_{n}^{(s)}(x, y ; \lambda)= \begin{cases}0, & \text { when } n=0, \\
\sum_{k=2 m+1}^{n} \sum_{m=0}^{\left[\frac{k-1}{2}\right]}\left(\begin{array}{l}
n \\
k
\end{array}\right) D_{n-k}(x ; \lambda)(-1)^{m} y^{2 m+1} \lambda^{k} \mathbb{S}_{1}(k, 2 m+1), & \text { when } n>0 .\end{cases}
\end{aligned}
$$


Proof. Proof is omitted since it is similarly obtained as shown in the proof of Theorem 10 by replacing $\frac{2}{2+\frac{1}{\lambda} \log (1+\lambda t)}$ by $\frac{\log (1+\lambda \log (1+t))}{\log (1+\lambda t)}$.

Theorem 15. For $n \geq 0$ and $r \in \mathbb{R}$, we have that

$$
\begin{aligned}
& \left.D_{n}^{(c)}(x+r, y ; \lambda)=\sum_{k=0}^{n} \sum_{l=0}^{k}\left(\begin{array}{l}
n \\
k
\end{array}\right) D_{n-k}^{(c)}(x, y ; \lambda)(r)\right)_{l} \lambda^{k-l} \mathbb{S}_{1}(k, l), \quad n \geq 0, \\
& \left.D_{n}^{(s)}(x+r, y ; \lambda)=\sum_{k=0}^{n} \sum_{l=0}^{k}\left(\begin{array}{l}
n \\
k
\end{array}\right) D_{n-k}^{(c)}(x, y ; \lambda)(r)\right)_{l} \lambda^{k-l} \mathbb{S}_{1}(k, l), \quad n \geq 1 .
\end{aligned}
$$

Proof. One can easily prove by replacing $C h_{n}^{(c)}(x, y ; \lambda)$ and $\frac{2}{2+\frac{1}{\lambda} \log (1+\lambda t)}$ by $D_{n}^{(c)}(x, y ; \lambda)$ and $\frac{\log (1+\lambda \log (1+t))}{\log (1+\lambda t)}$, respectively in the proof of Theorem 11 .

Theorem 16. For $n \in \mathbb{N}, D_{n}^{(c)}(x, y ; \lambda)$ and $D_{n}^{(s)}(x, y ; \lambda)$ satisfy the following expressions:

$$
\begin{aligned}
& D_{n}^{(c)}(x+1, y ; \lambda)-D_{n}^{(c)}(x, y ; \lambda)=\sum_{k=1}^{n}\left(\begin{array}{l}
n \\
k
\end{array}\right)\left(\lambda^{k-1} D_{n-k}^{(c)}(x, y ; \lambda) \mathbb{S}_{1}(k, 1)\right), \\
& D_{n}^{(s)}(x+1, y ; \lambda)-D_{n}^{(s)}(x, y ; \lambda)=\sum_{k=1}^{n}\left(\begin{array}{l}
n \\
k
\end{array}\right)\left(\lambda^{k-1} D_{n-k}^{(s)}(x, y ; \lambda) \mathbb{S}_{1}(k, 1)\right) .
\end{aligned}
$$

Proof. Noting that $\frac{1}{\lambda} \log (1+\lambda t)=\sum_{k=1}^{\infty} \lambda^{k-1} \mathbb{S}_{1}(k, 1) \frac{t^{k}}{k !}$, we have

$$
\begin{aligned}
\sum_{n=0}^{\infty} D_{n}^{(c)}(x+1, y ; \lambda) \frac{t^{n}}{n !} & =\frac{\log (1+\lambda \log (1+t))}{\log (1+\lambda t)}\left(1+\frac{1}{\lambda} \log (1+\lambda t)\right)^{x+1} \cos _{\lambda}^{(y)}(t) \\
& =\frac{\log (1+\lambda \log (1+t))}{\log (1+\lambda t)}\left(1+\frac{1}{\lambda} \log (1+\lambda t)\right)^{x}\left(1+\frac{1}{\lambda} \log (1+\lambda t)\right) \cos _{\lambda}^{(y)}(t) \\
& =\sum_{n=0}^{\infty} D_{n}^{(c)}(x, y ; \lambda) \frac{t^{n}}{n !}\left(1+\sum_{k=1}^{\infty} \lambda^{k-1} \mathbb{S}_{1}(k, 1) \frac{t^{k}}{k !}\right) \\
& =\sum_{n=0}^{\infty}\left(D_{n}^{(c)}(x, y ; \lambda)\right) \frac{t^{n}}{n !}+\sum_{n=1}^{\infty}\left(\sum_{k=1}^{n}\left(\begin{array}{l}
n \\
k
\end{array}\right) D_{n-k}^{(c)}(x, y ; \lambda) \lambda^{k-1} \mathbb{S}_{1}(k, 1)\right) \frac{t^{n}}{n !}
\end{aligned}
$$

which imply the first identity by comparing coefficients. Similarly, the second one is obtained from that

$$
\begin{aligned}
\sum_{n=0}^{\infty} D_{n}^{(s)}(x+1, y ; \lambda) \frac{t^{n}}{n !} & =\frac{\log (1+\lambda \log (1+t))}{\log (1+\lambda t)}\left(1+\frac{1}{\lambda} \log (1+\lambda t)\right)^{x+1} \sin _{\lambda}^{(y)}(t) \\
& =\sum_{n=1}^{\infty}\left(D_{n}^{(s)}(x, y ; \lambda)+\sum_{k=1}^{n}\left(\begin{array}{l}
n \\
k
\end{array}\right) D_{n-k}^{(s)}(x, y ; \lambda) \lambda^{k-1} \mathbb{S}_{1}(k, 1)\right) \frac{t^{n}}{n !}
\end{aligned}
$$

Corollary 3. For $n \in \mathbb{N}$, the following are satisfied that

$$
D_{n}(z+1 ; \lambda)-D_{n}(z ; \lambda)=\sum_{k=1}^{n}\left(\begin{array}{l}
n \\
k
\end{array}\right)\left(\lambda^{k-1} D_{n-k}(z ; \lambda) \mathbb{S}_{1}(k, 1)\right) .
$$




\section{Conclusions}

We study here some properties of Sheffer sequences of complex Changhee and Daehee polynomials by splitting them into real and imaginary parts using cosine- and sine-Sheffer sequences, (see Equations (6) and (22)). With the help of simplified trigonometric polynomial sequences, $C_{n}(z)$ and $S_{n}(z)$, which are defined in Equation (11), we give several expressions and properties (see Theorems 1-7) of the cosine- and sine-Sheffer sequences for complex Changhee and Daehee polynomials. Further, we have studied degenerate types (Definitions 29 and 39) of Sheffer sequences of complex Changhee and Daehee polynomials based on umbral composition, which are asymptotically extended to Sheffer sequences of the complex Changhee and Daehee polynomials as a limit of degenerate types when parameter $\lambda$ goes to 0 , and present some identities, properties (Theorems 8-12) and examples of the degenerate complex polynomials. In order to obtain useful identities and relations of them, we compare coefficients of infinite polynomial series of the generating functions by using the binomial convolution of sequences. In conclusion, we only study some properties of complex Changhee and Daehee polynomials as examples of Sheffer sequences, but one can apply this study to any type of Sheffer sequence. As is well known, the generalized Appell polynomials are one of the famous examples of Sheffer sequences and hence the most presented results can be applied to the generalized Appell polynomials.

Funding: This work was supported by the National Research Foundation of Korea (NRF) grant funded by the Korea government (MSIT) (No. 2019R1C1C1003869).

Conflicts of Interest: The author declares no conflict of interest.

\section{References}

1. Blasiack, P.; Dattoli, G.; Horzela, H.; Penson, K. Representations of monomiality principle with Sheffer-type polynomials and boson normal ordering. Phys. Lett. A 2006, 352, 7-12. [CrossRef]

2. Cesarano, C. Generalized special functions in the description of fractional diffusive equations. Commun. Appl. Ind. Math. 2019, 10, $31-40$. [CrossRef]

3. Cesarano, C. Multi-dimensional Chebyshev polynomials: A non-conventional approach. Commun. Appl. Ind. Math. 2019, 10, 1-19. [CrossRef]

4. Cesarano, C.; Ricci, P.E. The Legendre polynomials as a basis for Bessel functions. Int. J. Pure Appl. Math. 2016, 111, 129-139. [CrossRef]

5. Kim, D.S.; Kim, T.; Kwon, H.I.; Mansour, T. Powers under umbral composition and degeneration for Sheffer sequences. Adv. Diff. Equ. 2016, 2016, 66. [CrossRef]

6. Kim, T.; Kim, D.S.; Mansour, T.; Rim, S.-H.; Schork, M. Umbral calculus and Sheffer sequences of polynomials. J. Math. Phys. 2013, 54, 1-20. [CrossRef]

7. Roman, S. The Umbral Calculus, Pure and Applied Mathematics; Academic Press. Inc. [Harcourt Brace Jovanovich, Publishers]: New York, NY, USA, 1984; Volume 111, x+193 p, ISBN 0-12-594380-6.

8. Ruiz, J.A. Riordan arrays from an umbral symbolic viewpoint. Bol. Soc. Port. Mat. 2012, 67, 5-8.

9. Yasmin, G.; Muhyi, A.; Araci, S. Certain Results of q-Sheffer-Appell Polynomials. Symmetry 2019, 11, 159. [CrossRef]

10. Carlitz, L. Degenerate Stirling, Bernoulli and Eulerian numbers. Utilitas Math. 1979, 15, 51-88.

11. Carlitz, L. Some remarks on the Bell numbers. Fibonacci Quart. 1980, 18, 66-73.

12. Dolgy, D.V.; Kim, T. Some explicit formulas of degenerate Stirling numbers associated with the degenerate special numbers and polynomials. Proc. Jangjeon Math. Soc. 2018, 21, 309-317

13. Jang, G.-W.; Kim, T.; Kwon, H.-I. On the extension of degenerate Stirling polynomials of the second kind and degenerate Bell polynomials. Adv. Stud. Contemp. Math. (Kyungshang) 2018, 28, 305-316.

14. Kim, D. A Note on the Degenerate Type of Complex Appell Polynomials. Symmetry 2019, 11, 1339. [CrossRef]

15. Kim, D.S.; Kim, T. A note on polyexponential and unipoly functions. Russ. J. Math. Phys. 2019, 26, 40-49. [CrossRef]

16. Kim, D.S.; Kim, T.; Lee, H. A note on degenerate Euler and Bernoulli polynomials of complex variable. Symmetry 2019, 11, 1168. [CrossRef] 
17. Kim, T. A note on degenerate Stirling polynomials of the second kind. Proc. Jangjeon Math. Soc. 2017, 20, 319-331.

18. Kim, T.; Jang, G.-W. A note on degenerate gamma function and degenerate Stirling number of the second kind. Adv. Stud. Contemp. Math. (Kyungshang) 2018, 28, 207-214.

19. Kim, T.; Kim, D.S. Degenerate Laplace transform and degenerate gamma function. Russ. J. Math. Phys. 2017, 24, 241-248. [CrossRef]

20. Kim, T.; Kim, D.S. Degenerate central Bell numbers and polynomials. Rev. R. Acad. Clenc. Exactas Fís. Nat. Ser. A Mat. RACSAM 2019, 113, 2507-2513. [CrossRef]

21. Kim, T.; Kim, D.S.; Kwon, H.-I. A note on degenerate Stirlingnumbers and their applications. Proc. Jangjeon Math. Soc. 2018, 21, 195-203.

22. Kim, T.;Yao, Y.; Kim, D.S.; Jang, G.-W. Degenerate $r$-Stirlingnumbers and $r$-Bell polynomials. Russ. J. Math. Phys. 2018, 25, 44-58. [CrossRef]

23. Lee, J.G.; Kwon, J. The modified degenerate $q$-Bernoulli polynomials arising from $p$-adic invariant integral on $Z_{p}$. Adv. Diff. Equ. 2017, 29. [CrossRef]

24. Lee, J.G.; Jang, L.-C. On modified degenerate Carlitz $q$-Bernoulli numbers and polynomials. Adv. Diff. Equ. 2017, 22. [CrossRef]

25. Pyo, S.-S. Degenerate Cauchy numbers and polynomials of the fourth kind. Adv. Stud. Contemp. Math. (Kyungshang) 2018, 28, 127-138.

26. Pyo, S.-S.; Kim, T.; Rim, S.-H. Degenerate Daehee Numbers of the Third Kind. Mathematics 2018, 6, 239. [CrossRef]

27. Upadhyaya, L.M. On the degenerate Laplace transform IV. Int. J. Eng. Sci. Res. 2018, 6, 198-209.

28. Simsek, Y. Identities on the Changhee numbers and Apostol-type Daehee polynomials. Adv. Stud. Contemp. Math. (Kyungshang) 2017, 27, 199-212.

29. Jeong, J.; Kang, D.-J.; Rim, S.-H. Symmetry Identities of Changhee Polynomials of Type Two. Symmetry 2018, 10, 740. [CrossRef]

30. El-Desouky, B.S.; Mustafa, A. New Results and Matrix Representation for Daehee and Bernoulli Numbers and Polynomials. Appl. Math. Sci. 2015, 9, 3593-3610. [CrossRef]

31. He, Y.; Zhang, W. A three-term reciprocity formula for Bernoulli polynomials. Util. Math. 2016, 100, $23-31$.

32. Kim, D.S.; Kim, T. Daehee Numbers and Polynomials. Appl. Math. Sci. 2013, 7, 5969-5976, doi:10.12988/ams.2013.39535. [CrossRef] 